\title{
CONSTRUCTION OF OPEN SETS OF FREE $k$-TUPLES OF MATRICES
}

\author{
by A. MANDEL and J. Z. GONÇALVES*
}

(Received 10th August 1985)

\section{Introduction}

A $k$-tuple of members of a group is free if it freely generates a free group. In an earlier paper [1] the authors have shown:

Theorem 0 . Let $F$ be a locally compact field with a nontrivial absolute value. Then the set of free $k$-tuples of $P G L(n, F)$ has nonvoid interior in $P G L(n, F)^{k}$, where the latter has the topology induced from that of $F$.

In the present work we elaborate on the proof of the theorem above, in order to describe an explicit construction for open sets of free $k$-tuples. For this purpose, it is more convenient to work in $G L(n, F)$ than $P G L(n, F)$. The first step produces an open subset of $F^{k n^{2}+k n}$ and an open map $\dot{\phi}_{k}$ of it into $G L(n, F)^{k}$ whose image consists of free $k$-tuples; this, together with elementary facts about the quotient map $G L(n, F) \rightarrow P G L(n, F)$ yields Theorem 0 . The second step consists of intersecting that set with a suitable affine copy of $F^{n k^{2}}$ such that $\phi_{k}$ immerses that intersection into $G L(n, F)^{k}$. This provides open subsets of free $k$-tuples of $G L(n, F)$ described by exactly $k n^{2}$ parameters. It is not very hard to follow the computations in the proof on small examples over familiar fields; two such examples are included in the paper.

We introduce some notation. Throughout, $F$ is a locally compact field with a nontrivial absolute value $x \mapsto|x|$. A product space $F^{m}$ will be equipped with the norm $\left|\left(x_{1}, \ldots, x_{m}\right)\right|=\max \left\{\left|x_{1}\right|, \ldots,\left|x_{m}\right|\right\}$. As $F$ is fixed, we use the simple notation $M_{n}=M_{n}(F)$, $G L_{n}=G L_{n}(F)$ and denote by $D_{n}$ the set of diagonal matrices in $M_{n}$, and $N_{n}$ the subset of $D_{n}$ whose members have diagonal entries which are distinct and nonzero. We identify $M_{n}=F^{n^{2}}$ and $D_{n}=F^{n}\left(\right.$ via $\left.\left(x_{1}, \ldots, x_{n}\right) \mapsto \operatorname{diag}\left(x_{1}, \ldots, x_{n}\right)\right)$. Hence $G L_{n} \times N_{n}$ is an open and dense subset of $F^{n^{2}+n}$. Now we state our main results. Let $k$ be a positive integer and define the map

$$
\phi_{k}: G L_{n}^{k} \times D_{n}^{k} \rightarrow M_{n}^{k}
$$

by

$$
\phi_{k}\left(\left(A^{(1)}, \ldots, A^{(k)}\right),\left(D^{(1)}, \ldots, D^{(k)}\right)\right)=\left(A^{(1)} D^{(1)}\left(A^{(1)}\right)^{-1}, \ldots, A^{(k)} D^{(k)}\left(A^{(k)}\right)^{-1}\right)
$$

*This paper forms part of the Procedings of the conference Groups-St Andrews 1985. 
Theorem 1. The restriction of $\phi_{k}$ to $G L_{n}^{k} \times N_{n}^{k}$ is an open map.

An $m$-tuple $(m \geqq n)$ of members of $F^{n}$ is said to be in general position provided each $n$ of its members comprises a basis of $F^{n}$.

Theorem 2. Let $W=\left(W^{(1)}, \ldots, W^{(k)}\right) \in G L_{n}^{k}$. Suppose that the $k n$-tuple of columns of $W$ in general position. Then there exist a neighborhood $\mathscr{A}$ of $W$ in $G L_{n}^{k}$ and a subset $\mathscr{D}$ of $D_{n}^{k}$ with nonemplty interior such that $\phi_{k}(\mathscr{A} \times \mathscr{D})$ consists only of free $k$-tuples.

Note that as $N_{n}^{k}$ is dense in $D_{n}^{k}$, the combination of these two results yields Theorem 0 . As in [1], the discovery of Theorem 2 as well as many ideas in its proof are inspired by Tits [5].

One can remove the degeneracy of $\phi_{k}$, by restricting this map to a suitable subspace. For convenience of explicit constructions, let $P \in G L_{n}^{k}$ be $\mathrm{a}^{-} k$-tuple of permutation matrices and denote by $M_{n}^{p}$ the affine subspace of $M_{n}^{k}$ which consists of $k$-tuples $\left(A^{(1)}, \ldots, A^{(k)}\right)$ such that for $i=1, \ldots, k, A^{(i)}$ has a 1 in each entry where $P^{(i)}$ has a 1 .

Theorem 3. Let $W, \mathscr{A}, \mathscr{D}$ be as in Theorem 2. Then there exist a $k$-tuple $P$ of permutation matrices and an open set $\mathscr{B}$ of $G L_{n}^{k}$ such that the restrictions of $\phi_{k}$ to $\left(\mathscr{B} \cap M_{n}^{p}\right) \times \mathscr{D}$ is an open immersion and $\phi_{k}\left(\left(\mathscr{B} \cap M_{n}^{p}\right) \times \mathscr{D}\right)=\phi_{k}(\dot{A} \times \mathscr{D})$.

It is clear that the quotient map $G L_{n} \rightarrow P G L_{n}$ maps free $k$-tuples to free $k$-tuples. As this map is open, the theorems above yield open sets of free $k$-tuples in $P G L_{n}$. Actually, the open sets constructed in the proof of Theorem 2 are unions of fibres of this quotient map.

The authors have conjectured [1] that free $k$-tuples form (alternatively, contain) a dense open set in $P G L_{n}^{k}$. It was pointed to us by D. Sullivan that the conjectures fail for $P G L(2, \mathbb{C})$; this may be extracted from [4] where the interior of the set of free $k$-tuples of $P S L(2, \mathbb{C})$ is characterized.

\section{The $\operatorname{map} \phi_{k}$}

Proof of Theorem 1. It is enough to show that $\phi=\phi_{1}$ is an open map, as $\phi_{k}=(\phi, \ldots, \phi)$. In order to prove that, we compute the differential and show that it is surjective at each point of $G L_{n} \times N_{n}$. The desired result is a standard consequence of the "inverse mapping theorem" when $F=\mathbb{R}$ or $\mathbb{C}$; for non-archimedean $F$, see the remark following the proof.

Let us fix a point $(A, D) \in G L_{n} \times N_{n}$ and write $d \phi$ for the differential of $\phi$ at $(A, D)$. Using well known differentiation rules, we obtain:

$$
d \phi(H, K)=H D A^{-1}-A D A^{-1} H A^{-1}+A K A^{-1}
$$

To show that $d \phi$ is surjective, we consider instead the linear map $T: M_{n} \times D_{n} \rightarrow M_{n}$ defined by $T(H, K)=A^{-1} d \phi(A H, K)$. Thus

$$
T(H, K)=H D-D H+K .
$$


Let $E_{i j}$ be the matrix which has a 1 in position $i j$ and 0 elsewhere. It is enough to show that each $E_{i j}$ is in the image of $T$. Suppose that $D=\operatorname{diag}\left(x_{1}, \ldots, x_{n}\right)$, with the $x_{i}$ 's distinct. Direct computations using (2) yield:

$$
T\left(\left(x_{j}-x_{i}\right)^{-1} E_{i j}, 0\right)=E_{i j},
$$

if $i \neq j$ and

$$
T\left(0, E_{i i}\right)=E_{i i}
$$

Remark. A differential calculus for non-archimedean fields has been developed by Schikhof [3] with results that validate the proof above in that case. However, there seems to be a mistake in the proof of Theorem 2.3 of [3]. This can be bypassed by noting that proofs of the inverse mapping theorem using the notion of "strong derivative", as in Nijenhuis [2] (we are grateful to Prof. E. L. Lima for pointing out this reference to us) remain valid in the non-archimedean setting. That extends the theorem at least to rational functions $F^{m} \rightarrow F^{n}$, which is enough for our purposes.

Now we remove the degeneracy of $\phi$. This is done by first characterizing its fibres. That, together with Theorem 1 enables one to recognize the triple $\left(G L_{n} \times N_{n}, \phi, \operatorname{Im} \phi\right)$ as a principal $G$-bundle, where $G$ is the group of monomial matrices in $G L_{n}$ (matrices with exactly one nonzero on each row and column). Then we proceed to the construction of local cross sections.

Lemma 1.1. Let $(A, D)$ and $(\bar{A}, \bar{D})$ be elements of $G L_{n} \times N_{n}$. Then $\phi(A, D)=\phi(\bar{A}, \bar{D})$ if and only if there exists a monomial matrix $M$ such that $\bar{A}=A M$ and $\bar{D}=M^{-1} D M$. Further, there exists a neighborhood of $(A, D)$ whose intersection with any fibre of $\phi$ has fixed D-component.

Proof. Clearly $\phi\left(A M, M^{-1} D M\right)=\phi(A, D)$ if $M$ is a monomial matrix. For the converse, suppose that $\phi(A, D)=\phi(\bar{A}, \bar{D})$. It follows that the diagonal entries of $D$ and $\bar{D}$ are the eigenvalues of $\phi(A, D)$. Since $D \in N_{n}$, there is a uniquely determined permutation matrix $P$ such that $P^{-1} D P=\bar{D}$. One concludes that $\phi\left(\bar{A} P^{-1}, D\right)=\phi(A, D)$. Hence, for $i=1, \ldots, n$, the $i$ th column of $A$, and that of $\bar{A} P^{-1}$ are eigenvectors of $\phi(A, D)$ corresponding to the same eigenvalue. As each eigenspace of $\phi(A, D)$ is 1-dimensional, each column of $\bar{A} P^{-1}$ is a (nonzero) scalar multiple of the corresponding column of $A$, whence $\bar{A} P^{-1}=A K$ for some diagonal matrix $K$. The result follows with $M=K P$.

As for the last assertion, we note first that the action of monomial matrices on $N_{n}$, given by conjugation, factors through the induced action of the symmetric group, hence has finite. orbits. Thus, given $(A, D)$ the assertion is validated by a product neighborhood $B \times C$, where $C$ is a ball in $N_{n}$ centred in $D$, of radius less than half the distance of $D$ to the finite set $\left\{P D P^{-1} \mid P\right.$ a non-identity permutation matrix $\}$.

Theorem 1.2. Let $G L_{n}^{I}$ be the subset of matrices in $G L_{n}$ whose main diagonal consists of ones only. Then the restriction of $\phi$ to $G L_{n}^{I} \times N_{n}$ is an open immersion onto $\phi\left(G L_{n} \times N_{n}\right)$. 
Proof. Let $(A, D) \in G L_{n}^{I} \times N_{n}$. Choose a neighborhood of $(A, D)$ in $G L_{n} \times N_{n}$ satisfying the conclusions of Lemma 1.1 , and let $B$ be its intersection with $G L_{b}^{I} \times N_{n}$. Suppose that $\left(A^{\prime}, D^{\prime}\right)$ and $\left(A^{\prime \prime}, D^{\prime \prime}\right) \in B$ have the same image under $\phi$. By choice of $B, D^{\prime} \rightarrow D^{\prime \prime}$, hence, by the Lemma, $A^{\prime \prime}=A^{\prime} K$, for some diagonal matrix $K$. Since $A^{\prime}$ and $A^{\prime \prime} \in G L_{n}^{\prime}, A^{\prime}=A^{\prime \prime}$, whence $\phi \mid B$ is $1-1$. It will now follow that $\phi \mid G L_{n}^{I} \times N_{n}$ is an immersion from the fact that it is open, proved as follows:

Let $G L_{n}^{\prime}$ be the open subset of $G L_{n}$ comprised of matrices with nonzero diagonal entries, and let $\xi: G L_{n}^{\prime} \rightarrow G L_{n}^{I}$ be division of each row by the corresponding diagonal element. With $l: G L_{n}^{\prime} \rightarrow G L_{n}^{\prime}$ denoting the inclusion, we have the following commutative diagram of maps:

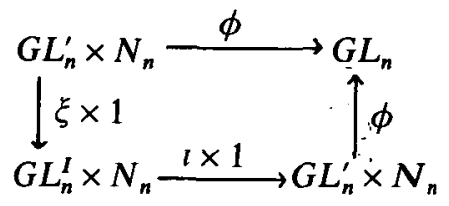

Since $\phi$ is open and $\xi$ is continuous, it follows that $\phi \circ l \times 1=\phi \mid G L_{n}^{I} \times N_{n}$ is open.

To see that $\phi\left(G L_{n}^{I} \times N_{n}\right)=\phi\left(G L_{n} \times N_{n}\right)$, choose $(A, D) \in G L_{n} \times N_{n}$; choose a nonzero term in the determinant expansion of $A$ and let $M$ be. the monomial matrix corresponding to that term. Then, $\left(A M^{-1}, M D M^{-1}\right) \in G L_{n}^{I} \times N_{n}$ and $\phi\left(A M^{-1}, M D M^{-1}\right)=$ $\phi(\mathrm{A}, \mathrm{D})$.

An obvious, but convenient consequence of this is stated here with the notation of Theorem 3.

Corollary 1.3. Let $P=\left(P^{(1)}, \ldots, P^{(k)}\right)$ be a $k$-tuple of permutation matrices. Then the restriction of $\phi_{k}$ to $\left(G L_{\ominus}^{P}\right)^{k} \times N_{n}^{k}$ is an open immersion onto $\phi_{k}\left(G L_{n}^{k} \times N_{n}^{k}\right)$.

\section{Construction of free $k$-tuples}

The proof of Theorem 2 is a modification of the proof of Proposition 2.12 of Tits [5]. As in [1], the tool for proving a $k$-tuple to be free is the following criterion, due to Tits, and which has as ancestor Klein's Table-tennis Lemma.

Lemma 2.1. Let $G$ be a group acting on a set $P$ and let $g=\left(g_{1}, \ldots, g_{k}\right)$ be a $k$-tuple of members of $G$. Suppose that there exists a k-tuple $\left(P_{1}, \ldots, P_{k}\right)$ of subsets of $P$ and $p \in P-\left(P_{1} \cup \cdots \cup P_{k}\right)$ such that, for all distinct $i, j, 1 \leqq i, j \leqq k$, and for all $n \in \mathbb{Z}^{*}$, $g_{i}^{n}\left(P_{j} \cup\{p\}\right) \subseteq P_{i}$. Then, $g$ is free.

This is the plan of the proof of Theorem 2. Beginning with the given $k$-tuple $W$, we choose a point $p \in F^{n}$ that will play the role suggested in Lemma 2.1. Next we choose suitable small disks in $F^{n}$, centred at the first and last column of each $W^{(i)}$ (for simplicity, all with the same radius); for each $i$, the union of all 1-dimensional subspaces through the two corresponding disks will yield the set $P_{i}$ of Lemma 2.1. Then the set $A$ is chosen as a convenient disk about $W$. Finally the set $\mathscr{D}$ is chosen subject to two requirements: for any $(A, D) \in \mathscr{A} \times \mathscr{D}$, the $k$-tuple $g=\phi_{k}(A, D)$ satisfies: 
(a) the first (last) column of each $A^{(i)}$ is an eigenvector corresponding to the largest (smallest) eigenvalue of $g_{i}$, and

(b) the relative sizes of the entries of members of $\mathscr{D}$ satisfy computed constraints that guarantee that $g_{i}\left(P_{j} \cup\{p\}\right)$ and $g_{i}^{-1}\left(P_{j} \cup\{p\}\right)$ are contained in $P_{i}, i \neq j$. A simple observation will then show that the conditions of Lemma 2.1 are fulfilled for each $g \in \phi(\mathscr{A} \times \mathscr{D})$. Note that the $P_{i}$ 's and $p$ are the same for all $g$ 's.

We need two simple preliminary results. Denote $F^{n}$ by $V$. Recall that when $m \geqq n$ an $m$-tuple of members of $V$ is in general position if every $n$ of its members are linearly independent.

Lemma 2.2. The set $G_{m}$ of $m$-tuples $(m \geqq n)$ of $V$ in general position is open in $V^{m}$.

Proof. Indeed, $\left(V_{1}, \ldots, V_{m}\right)$ is in general position if and only if for each $1 \leqq i_{1}<\cdots<i_{n} \leqq m, \operatorname{det}\left(V_{i_{1}}, \ldots, V_{i_{n}}\right) \neq 0$. That is, $G_{m}$ is the complement in $V^{m}=F^{n m}$ of a finite number of hypersurfaces.

If $A$ is a matrix (vector), $A_{i}$ denote its $i$ th column (component).

Lemma 2.3. Suppose that $A \in M_{n}$ and $x \in V$ are such that $\left(\dot{A_{1}}, \ldots, A_{n}, x\right) \in G_{n+1}$. Then, $A$ is nonsingular and each component of $A^{-1} x$ is nonzero.

Proof. That $A$ is nonsingular follows immediately from the definition of general position. Also, as $x=\Sigma_{i} A_{i}\left(A^{-1} x\right)_{i}$, general position of $\left(A_{\mathfrak{1}}, \ldots, A_{n}, x\right)$ implies that $\left(A^{-1} x\right)_{i} \neq 0$.

We have arrived at the objective of this section. Denote, whenever a set $S \subseteq F^{r}$ (some $r$ ) and $\alpha$ is a positive real, by

$$
\mathscr{B}(S, \alpha)=\left\{x \in F^{r} \mid d(s, x) \leqq \alpha \text { for some } s \in S\right]
$$

the closed $\alpha$-neighborhood of $S$.

Proof of Theorem 2. Regarding $W$ as a list of column vectors, by hypothesis $W \in G_{n k}$. Choose a point $p \in V$ not lying on any of the hyperplanes spanned by columns of $W$; thus $w=(W, p) \in G_{n k+1}$. Choose now positive reals $\gamma, \varepsilon, \delta$ such that:

(a) $\mathscr{B}(w, \gamma) \subseteq G_{n k+1}$ (this is possible, by Lemma 2.2),

(b) $\mathscr{B}(\mathscr{B}(w, \delta), \varepsilon) \subseteq \mathscr{B}(w, \gamma)$ (clearly, $\delta+\varepsilon \leqq \gamma$ suffices but in working on nonarchimedean examples, $\gamma=\varepsilon=\delta$ will also do). now:

Define now, for $i=1, \ldots, k, B_{s}=\mathscr{B}\left(W_{1}^{(s)}, \gamma\right), C_{i}=\mathscr{B}\left(W_{n}^{(i)}, \gamma\right)$ and $\mathscr{A}^{(i)}=\mathscr{B}\left(W^{(i)}, \delta\right)$. Set

(c) $\mathscr{A}=\mathscr{B}(W, \delta)=\mathscr{A}^{(1)} \times \cdots \times \mathscr{A}^{(k)}$,

(d) $P_{i}=\left\{x \in V \mid \lambda x \in B_{i} \cup C_{i}\right.$ for some $\left.\lambda \in F\right\}$. 
Finally, we define $\mathscr{D}=\mathscr{D}^{(1)} \times \cdots \times \mathscr{D}^{(k)}$, where

(e) $\mathscr{D}^{(i)}=\left\{\operatorname{diag}\left(\lambda_{1}, \ldots, \lambda_{n}\right)\left|\mu_{i}^{-1}\right| \lambda_{1}|\geqq| \lambda_{2}|\geqq \cdots \geqq| \lambda_{n-1}\left|\geqq \mu_{i}\right| \lambda_{n} \mid>0\right\}$, where $\mu_{1}, \ldots, \mu_{k}$ are positive reals yet to be determined. To begin with, we just require that $\mu_{i} \geqq 1$. The other restrictions on $\mu_{i}$ will be determined so that the following is true.

(f) If $g=\left(g_{1}, \ldots, g_{k}\right) \in \phi_{k}(\mathscr{A} \times \mathscr{B})$, then for $1 \leqq i, j \leqq k, i \neq j$, and $x \in P_{j} \cup\{p\}$, there exist $\alpha, \alpha^{\prime} \in F^{*}$ such that $g_{i}(\alpha x) \in B_{i}$ and $g_{i}^{-1}\left(\alpha^{\prime} x\right) \in C_{i}$.

Let us finish the proof of the Theorem under the assumption that (f) holds. We must show that each $g \in \phi_{k}(\mathscr{A} \times \mathscr{D})$ is free. Now, from (f) and the definition of the $P_{i}$, it follows clearly that $g_{i}^{ \pm 1}\left(P_{j} \cup\{p\}\right) \subseteq P_{i}$, whenever $i \neq j$. Also, since $\mu_{i} \geqq 1$, if $D \in \mathscr{D}^{(i)}$, so does $D^{r}$ for every positive $r$ hence $\left(g_{i}^{r}, \ldots, g_{k}^{r}\right) \in \phi_{k}(\mathscr{A} \times \mathscr{D})$ for each positive $r$. Applying the previous observation to $\left(g_{1}^{r}, \ldots, g_{k}^{r}\right), r>0$, shows that the conditions of Lemma 2.2 are fulfilled.

Incidentally, note that if some $\mu_{i}=1$, we could obtain $g_{i}=I$, which cannot be a member of a free $k$-tuple. This shows that if the numbers $\mu_{i} \geqq 1$ are such that (f) holds, indeed $\mu_{i}>1, i=1, \ldots, k$.

Now we compute the $\mu_{i}$ 's. Let us denote, for $r=1$ or $n$,

$$
\mathscr{L}_{r}=\left\{\operatorname{diag}\left(\lambda_{1}, \ldots, \lambda_{n}\right)\left|\lambda_{r}=0,\right| \lambda_{i} \mid \leqq 1 \text { for } i=1, \ldots, n\right\} \subseteq D_{n} .
$$

Now, for $1 \leqq i, j \leqq k, i \neq j$ and $r=1$ or $n$, let

$$
\beta_{i, j, r}=\sup \left\{\left\|\left(A D A^{-1} x\right) /\left(A^{-1} x\right)_{r}\right\| \mid A \in \mathscr{A}^{(i)}, x \in B_{j} \cup C_{j} \cup\{p\}, D \in \mathscr{L}_{r}\right\} .
$$

This is finite: when $A, x$ are the domain of this expression, the columns of $A$ occur together with $x$ in a member of $G_{n k+1}$ (see (a)), hence by Lemma 2.3 the denominator of $\left\|\left(A D A^{-1} x\right) /\left(A^{-1} x\right)_{r}\right\|$ never vanishes, and this is a continuous real valued function with compact domain.

Set now

$$
\mu_{i}=\sup _{j \neq i}\left\{\frac{\beta_{i, j, 1}}{\varepsilon}, \frac{\beta_{i, j, n}}{\varepsilon}, 1\right\}
$$

(As remarked before, it will follow that $\mu_{i}>1$; hence the presence of 1 in the expression defining $\mu_{i}$ is unnecessary. But we know no other "a priori" way of guaranteeing that $\mu_{i} \geqq 1$ ).

Now we prove (f). Let $g_{i}=A D A^{-1}$, with $A \in \mathscr{A}^{(i)}, D \in \mathscr{D}^{(i)}$, and let $x \in P_{j} \cup\{p\}$. Let $\alpha=\left(D_{11},\left(A^{-1} x\right)_{1}\right)^{-1}$. Then

$$
g_{i}(\alpha x)=\left(A D A^{-1} x\right) /\left(D_{11}\left(A^{-1} x\right)_{1}\right)=A \bar{D} A^{-1} \bar{x},
$$

where

$$
\bar{D}=D_{11}^{-1} D=\operatorname{diag}\left(1, \lambda_{2}, \ldots, \lambda_{n}\right), \lambda_{i}=D_{i i} / D_{1 i}
$$

and $\bar{x}=x /\left(A^{-1} x\right)_{1}$. Note that $\mu_{i}^{-1}>\left|\lambda_{2}\right| \geqq\left|\lambda_{3}\right| \geqq \cdots \geqq\left|\lambda_{n}\right|$. Further, $\left(A^{-1} \bar{x}\right)_{1}=1$ whence 
the identity $A_{1}=A \operatorname{diag}(1,0, \ldots, 0) A^{-1} \bar{x}$. We have:

$$
\left\|g_{i}(\alpha x)-A_{1}\right\|=\left\|A \bar{D} A^{-1} \bar{x}-A \operatorname{diag}(1,0, \ldots, 0) A^{-1} \bar{x}\right\|=\left\|\lambda_{2} A \bar{D} A^{-1} \cdot \bar{x}\right\|,
$$

where $\overline{\bar{D}}=\lambda_{2}^{-1} \operatorname{diag}\left(0, \lambda_{2}, \ldots, \lambda_{n}\right) \in \mathscr{L}_{1}$, yielding finally

$$
\left\|g_{i}(\alpha x)-A_{1}\right\|=\left|\lambda_{2}\right|\left\|\left(A \overline{\bar{D}} A^{-1} x\right) /\left(A^{-1} x\right)_{1}\right\| \leqq \mu_{i}^{-1} \beta_{i, j, 1} \leqq \varepsilon .
$$

Therefore as $A \in \mathscr{A}^{(i)}, A_{i} \in \mathscr{B}\left(W_{1}^{(i)}, \delta\right)$ whence

$$
g_{i}(\alpha x) \in \mathscr{B}\left(\mathscr{B}\left(W_{1}^{(i)}, \delta\right), \varepsilon\right) \subseteq B_{i} .
$$

This completes the proof of the conclusion of (f) about $g_{i}$; for $g_{i}^{-1}$, use the same argument with $\alpha^{\prime}=D_{n n} /\left(A^{-1} x\right)_{n}$ and replace $A_{1}, W_{1}^{(i)}$ by $A_{n}, W_{n}^{(i)}$.

\section{Examples}

We shall follow here the proof of Theorem 2 in order to construct examples of open sets of free pairs, first over the reals, second over $\mathbb{Q}_{p}$, the $p$-adic completion of the field of rational numbers. The simplification suggested by Corollary 1.3 is to be followed so that we obtain an 8-parameter family of free pairs, where each parameter is allowed to vary within a certain interval. A computation of appropriately sized intervals, which ensure that the resulting pairs are free is the gist of the calculation.

3.1. Let $F=\mathbb{R}, n=k=2$

$$
W=\left(\begin{array}{cc:cc}
1 & 0 & 1 / 2 & 1 \\
0 & 1 & 1 & 1 / 2
\end{array}\right)
$$

$p=(1,1), \gamma=1 / 6$. One readily verifies that $B(W, 1 / 6) \subseteq G_{5}$. In the notation of Corollary 1.3 , with $\xi$ suitably adapted to $P$,

$$
P=\left(\begin{array}{ll|ll}
1 & 0 & 0 & 1 \\
0 & 1 & 1 & 0
\end{array}\right), \xi(B(W, 1 / 6))=\left(\begin{array}{cc|cc}
1 & x_{2} & 1 / 2+x_{3} & 1 \\
x_{1} & 1 & 1 & 1 / 2+x_{4}
\end{array}\right)
$$

where $\left|x_{1}\right|,\left|x_{2}\right| \leqq 1 / 5,-\frac{3}{14} \leqq x_{3}, x_{4} \leqq \frac{3}{10}$. Recall that $\xi\left(B_{1}\right), \xi\left(C_{1}\right), \xi\left(B_{2}\right), \xi\left(C_{2}\right)$ are the projections of $\xi(B(W, 1 / 6))$ onto the first through fourth column, respectively.

Choosing $\delta=1 / 11, \varepsilon=5 / 66$ (so that $\varepsilon+\delta=1 / 6$ ), we have, with $\mathscr{A} \sim B(W, \delta)$,

$$
\xi(\mathscr{A})=\left(\begin{array}{cc:cc}
1 & \varepsilon_{2} & 1 / 2+\varepsilon_{3} & 1 \\
\varepsilon_{1} & 1 & 1 & 1 / 2+\varepsilon_{4}
\end{array}\right) \begin{aligned}
& \left|\varepsilon_{1}\right|,\left|\varepsilon_{2}\right| \leqq 1 / 10, \\
& -1 / 8 \leqq \varepsilon_{2}, \varepsilon_{4} \leqq 3 / 20
\end{aligned}
$$

Note that $\mathscr{L}_{1}=\left\{\lambda E_{22}|| \lambda \mid \leqq 1\right\}$. 
If $A \in \xi\left(\mathscr{A}^{(1)}\right)$,

$$
A=\left(\begin{array}{cc}
1 & \varepsilon_{2} \\
\varepsilon_{1} & 1
\end{array}\right),(\operatorname{det} A) \cdot A^{-1}=\left(\begin{array}{cc}
1 & -\varepsilon_{2} \\
-\varepsilon_{1} & 1
\end{array}\right),(\operatorname{det} A) \cdot A E_{22} A^{-1}=\left(\begin{array}{cc}
-\varepsilon_{1} \varepsilon_{2} & \varepsilon_{2} \\
\varepsilon_{1} & 1
\end{array}\right)
$$

If $x \in B_{2}$,

$$
\operatorname{det} A \cdot A E_{22} A^{-1} x=\left(\varepsilon_{2}\left(-\varepsilon_{1}\left(1 / 2+x_{3}\right)+1\right), \varepsilon_{1}\left(1 / 2+x_{3}\right)+1\right) \text {, }
$$

hence

$$
\left|(\operatorname{det} A) \cdot A E_{22} A^{-1} x\right| \leqq 1 / 2(1 / 2+3 / 10)+1=\frac{108}{100}
$$

also

$$
\left|\operatorname{det}(A) \cdot\left(A^{-1} x\right)_{1}\right|=\left|\frac{1}{2}+x_{3}-\varepsilon_{2}\right| \geqq \frac{1}{2}-\frac{3}{14}-\frac{1}{10}=\frac{13}{70} .
$$

Hence

$$
\sup \left\{\left|\frac{A D A^{-1} x}{\left(A^{-1} x\right)_{1}}\right| \mid A \in \xi\left(\mathscr{A}^{(1)}\right), D \in \mathscr{L}_{1}, x \in B_{2}\right\} \leqq \frac{108}{100} / \frac{13}{70}=\frac{378}{65}
$$

(A slightly more complicated exact calculation gives $\sup =\frac{72}{13}$ ).

Similarly, one finds that

$$
\sup \left|\frac{A D A^{-1} x}{\left(A^{-1} x\right)_{1}}\right|
$$

is bounded above by $90 / 92$ when $x \in C_{2}$, and by $11 / 9$ when $x=p$.

It follows that $\beta_{1,2,1} \leqq 378 / 65$, and the same bound holds for $\beta_{1,2,2}$ (no coincidencethis reflects symmetries of the chosen set $\mathscr{A}$ ).

Thus one gets

$$
\mu_{1}=\left\{\frac{b_{1,2,1}}{\varepsilon}, \frac{\beta_{1,2,2}}{\varepsilon}, 1\right\} \leqq \frac{24948}{325}<77
$$

Similar calculations yield $\mu_{2}=\frac{14916}{175}<86$ (additional effort yields the exact values $\left.\mu_{1}=4752 / 65, \mu_{2}=407 / 5\right)$.

In conclusion, Theorem 2 guarantees that,

$$
\phi_{2}\left(A^{(1)}, A^{(2)}, D^{(1)}, D^{(2)}\right)
$$

is a free pair whenever

$$
A^{(1)}=\left(\begin{array}{cc}
1 & \varepsilon_{2} \\
\varepsilon_{1} & 1
\end{array}\right),\left|\varepsilon_{1}\right|,\left|\varepsilon_{2}\right| \leqq 1 / 10
$$




$$
\begin{aligned}
& A^{(2)}=\left(\begin{array}{cc}
1 / 2+\varepsilon_{2} & 1 \\
1 & 1 / 2+\varepsilon_{4}
\end{array}\right),-1 / 8 \leqq \varepsilon_{3}, \varepsilon_{4} \leqq 3 / 20 \\
& D^{(1)}=\left(\begin{array}{cc}
\alpha_{1} & 0 \\
0 & \alpha_{2}
\end{array}\right) ;\left|\alpha_{1}\right|>\mu_{1},\left|\alpha_{2}\right|>0 \\
& D^{(2)}=\left(\begin{array}{cc}
b_{1} & 0 \\
0 & \beta_{2}
\end{array}\right) ;\left|\beta_{1}\right|>\mu_{2},\left|\beta_{2}\right|>0
\end{aligned}
$$

and by Corollary 1.3 the image of the interior of this domain is an open set.

\subsection{Let $F=\mathbb{Q}_{P}$}

We indicate one set of parameters; the calculations, following the first example as paradigm, are easily completed due to the ultrametric property of $\|_{P}$, yielding exact values for $\mu_{1}$ and $\mu$

Let

$$
W=\left(\begin{array}{ll:ll}
1 & 0 & P & 1 \\
0 & 1 & 1 & P
\end{array}\right), P=(1,1),
$$

with $\gamma=P^{-2}$. We take $\varepsilon=\delta=\gamma$. Then

$$
\xi(B(W), \gamma)=\xi(\mathscr{A})=\left(\begin{array}{cc:cc}
1 & \varepsilon_{2} & P+\varepsilon_{3} & 1 \\
\varepsilon_{1} & 1 & 1 & P+\varepsilon_{4}
\end{array}\right),\left|\varepsilon_{i}\right|_{P} \leqq P^{2}
$$

One gets $\mu_{1}=\mu_{2}=P^{3}$. Thus one gets an open set of free pairs of $P G L\left(2, \mathbb{Q}_{P}\right)$ as $\phi_{2}\left(A^{(1)}, A^{(2)}, D^{(1)} D^{(2)}\right)$ (sort of) where

$$
\begin{aligned}
& A^{(1)}=\left(\begin{array}{cc}
1 & P^{2} d_{2} \\
P^{2} d_{1} & 1
\end{array}\right), A^{(2)}=\left(\begin{array}{cc}
P+P^{2} d_{3} & 1 \\
1 & P+P^{2} d_{4}
\end{array}\right)\left|d_{i}\right|_{P} \leqq 1,1 \leqq i \leqq 6 . \\
& D^{(1)}=\left(\begin{array}{cc}
1 & 0 \\
0 & P^{2} d_{5}
\end{array}\right), D^{(2)}=\left(\begin{array}{cc}
1 & 0 \\
0 & P^{2} d_{6}
\end{array}\right) .
\end{aligned}
$$

\section{What about Zariski Topology}

It makes sense to ask whether an analogue of Theorem 0 exists in the sense of Zariski topology; namely, one would look for Zariski-open sets of free $k$-tuples. For fields with a non-trivial absolute value, those sets would be open and dense, in the topology hitherto considered. Thus, we would be looking for something even stronger than conjecture 2 of [1], which has been shown false for $\mathbb{C}$ by Sullivan, as we remarked at the introduction. Indeed, the intended analogue of Theorem 0 is false in general, as we show now by elementary methods. 
Here $F$ will be a field with infinitely many roots of unity. The only topology considered herewith in the several spaces involved is the Zariski topology ascribed to them by their obvious identification as a constructible subset of some $F^{m}$.

Theorem 4.1. The set $T_{n}=\left\{A \in G L_{n} \mid A^{s}=I\right.$ for some $\left.s \in \mathbb{N}^{*}\right\}$ is dense in $G L_{n}$.

Corollary 4.2. $T_{n}^{k}$ is dense in $G L_{n}^{k}$.

Corollary 4.3. The set of free $k$-tuples has empty interior in $G L_{n}^{k}$.

Since the product of dense sets is dense in the Zariski product topology, Theorem 4.1 implies the first corollary, and this entails the second one immediately. Notice that the existence of infinitely many roots of unity is also a necessary condition for Theorem 4.1 to hold: if a field has only $r$ roots of 1 , every member of $T_{n}$ is a root of the polynomial (in $n^{2}$ variables) $(\operatorname{det} A)^{r}-1$. However, one feels that Corollary 4.3 is true whenever $F$ is infinite.

The proof of 4.1 requires the two lemmas below:

Lemma 4.4. The set $T_{n}(F)$ is dense in the set of semisimple matrices of $G L(n, K)$, where $K$ is the algebraic closure of $F$.

Proof. Consider the morphism of varieties

$$
\phi: G L(n, K) \times D_{n}(K) \rightarrow G L(n, K),
$$

given by the now usual formula $\phi(A, D)=A D A^{-1}$. Its image is the set of semisimple matrices. The set $T_{n}(F)$ contains the image of $G L(n, F) \times D_{n}(R)$, where $R$ is the set of roots of 1 in $F, D_{n}(R)$ is the set of diagonal matrices all whose diagonal entries are in $R$. Since $R$ is infinite, it is dense in $K$, hence $D_{n}(R)$ is dense in $D_{n}(K)$; essentially by the same token $G L(n, F)$ is dense in $G L(n, K)$. It follows that $G L(n, K) \times D_{n}(R)$ is dense in the domain of $\phi$; by continuity, its image is dense in the image of $\phi$.

Lemma 4.3. For an algebraically closed field $K$, the set of matrices with only simple eigenvalues is open in $M_{n}(K)$.

Proof. Let $X=\left(x_{i j}\right)$ be a matrix of indeterminates over $R$ and let $d\left(x_{i j}\right) \in K\left[x_{i j}\right]$ be the discriminant of the characteristic polynomial $P_{X}$ of $X$. A polynomial has multiple roots if and only if its discriminant is zero. Hence a matrix $A$ has multiple eigenvalues if and only if it is a zero of $d\left(x_{i j}\right)$.

Proof of Theorem 4.1. Combining the two lemmas, $T_{n}(F)$ is dense in a subset of $G L(n, K)$ which contains an open set. As open sets are dense, $T_{n}(F)$ is dense in $G L(n, K)$; a fortiori, $T_{n}(F)$ is dense in $G L(n, F)$, since this has the topology induced from $G L(n, K)$. 


\section{REFERENCES}

1. A. Mandel and J. Z. Gonçalves, Free $k$-tuples in linear groups, Bull. Austr. Math. Soc. 28 (1983), 152-157.

2. A. Nuenhuis, Strong derivatives and inverse mappings, Amer. Math. Monthly 81 (1971), 969981 and 83 (1976), 22.

3. W. H. Schiкhof, Differentiation in non-archimedean valued fields, Nederl. Akad. Wetensch. Proc. Ser. A 73 (1970), 35-44.

4. D. Sullivan, Quasi conformal homeomorphisms and dynamics II: Structural stability implies hyperbolicity for Kleinian groups, preprint, 1982.

5. J. Tirs, Free subgroups in linear group, J. Algebra 20 (1972), 250-270.

Instituto de Matemática e Estatística

Universidade de São Paulo

Caixa Postal, 20.570-Ag. Iguatemi

01000-São Paulo-SP-Brazil 